\title{
Experimental Analysis of Approximation Algorithms for the Vertex Cover and Set Covering Problems ${ }^{2}$
}

\author{
Fernando C. Gomes ${ }^{\mathrm{a}}$, Cláudio N. Meneses ${ }^{\mathrm{b}, 1}$, Panos M. Pardalos ${ }^{\mathrm{b}, *}$, \\ Gerardo Valdisio R. Viana ${ }^{\mathrm{a}, \mathrm{c}}$ \\ ${ }^{a}$ Computer Science Department, Artificial Intelligence Laboratory, Federal University of Ceara, Brazil \\ ${ }^{\mathrm{b}}$ Department of Industrial and Systems Engineering, University of Florida, 303 Weil Hall, Gainesville, FL 32611, USA \\ ${ }^{\mathrm{c}}$ Computer Science Department, State University of Ceara, Brazil
}

Available online 2 February 2006

\begin{abstract}
Several approximation algorithms with proven performance guarantees have been proposed to find approximate solutions to classical combinatorial optimization problems. However, theoretical results may not reflect the experimental performance of the proposed algorithms. As a consequence, a question arises: how "far" from the theoretically proved performance are the experimental results? We conduct a controlled empirical study of approximation algorithms for the Vertex Cover and the Set Covering Problems. Many authors have proposed approximation algorithms for those problems. Our main goal is to better understand their strengths, weaknesses, and operation. Although we implement more than one algorithm to find feasible solutions to either problems, this work does not emphasize competition between them. The quality of the solutions related to the theoretical performance guarantees are analyzed instead. The computational experiments showed that the proven performance guarantees of all tested algorithms did not forecast well the empirical performance.
\end{abstract}

(c) 2005 Elsevier Ltd. All rights reserved.

Keywords: Approximation algorithms; Experimental analysis; Vertex Cover; Set covering

\footnotetext{
This work has been partially supported by NSF and CRDF grants.

* Corresponding author.

E-mail addresses: carvalho@lia.ufc.br (F.C. Gomes), claudio@ufl.edu (C.N. Meneses), pardalos@ufl.edu (P.M. Pardalos), valdisio@lia.ufc.br (G.V.R. Viana).

${ }^{1}$ Supported by the Brazilian Federal Agency for Higher Education (CAPES)—Grant No. 1797-99-9.
} 


\section{Introduction}

Several approximation algorithms with proven performance guarantees have been proposed to find approximate solutions to classical combinatorial optimization problems. However, theoretical results may not reflect the experimental performance of the proposed algorithms. As a consequence, a question arises: how "far" from the theoretically proved performance are the experimental results?

In this paper we conduct a controlled empirical study of algorithms with proven performance, which have been proposed to two classical combinatorial optimization problems, namely, the Vertex Cover Problem and the Set Covering Problem. Both problems are known to be NP-hard.

In order to provide a performance guarantee for a certain algorithm one must find an approximation factor $\rho$ that gives the worst-case distance to the optimum. A $\rho$-approximation algorithm for a maximization (minimization) problem $P$ is a polynomial time algorithm that provides a solution with a value that is at least (at most) $\rho$ times the value of an optimal solution to $P$. We note that for a maximization (minimization) problem $0 \leqslant \rho \leqslant 1(\rho \geqslant 1)$.

The purpose of this work is to test a broad range of instances of the Vertex Cover and Set Covering Problems using simple algorithms proposed in the literature, in order to evaluate the empirical performance and compare with their approximation factors. In doing so, one better understands algorithm's strengths, weaknesses, and operation. Moreover, we are concerned with general instances of the problems, rather than specific ones. The empirical performance is measured through quality of solutions, computational effort, and instance size.

In Grossman and Wool [1], conducted a comparative study of nine approximation algorithms for the Unweighted Set Covering Problem. This problem is a special case of the Set Covering Problem where all costs have value 1 . In our paper we use two of those nine algorithms, namely the Round and the Greedy algorithms. The reason we test again these two algorithms is that they had good empirical performance in that paper.

In performing the computational experiments we follow ideas described in the papers [2,3] how to report experimental analyses of heuristics.

The paper is organized as follows. The problems and algorithms are described in Section 2. In Section 3 experiment's set up and computational results are shown. In Section 4 we discuss the experimental results. In Section 5 concluding remarks are given.

\section{Problems and their approximation algorithms}

\subsection{Vertex Covering Problem}

A Vertex Cover is a set of nodes in a graph such that every edge of the graph has at least one endpoint in the set. A minimum cover is a Vertex Cover which has the smallest number of nodes for a given graph. In this paper we are concerned with both the Unweighted Vertex Cover and the Weighted Vertex Cover. In the former we are interested in finding a minimum cardinality Vertex Cover, while in the latter we ask for a cover of minimum total weight. Both problems have been used in a wide variety of real-world applications, such as crew scheduling [4], VLSI design [5], nurse rostering [6], and industrial machine 
Table 1

Approximation algorithms for the Vertex Cover Problem

\begin{tabular}{lll}
\hline Algorithm & $\begin{array}{l}\text { Performance } \\
\text { guarantee }\end{array}$ & Ref. \\
\hline Greedy & 2 & {$[8]$} \\
Round & 2 & {$[9,10]$} \\
Dual-LP & 2 & {$[11]$} \\
\hline
\end{tabular}

assignments [7]. Next we give formal definitions of the Unweighted Vertex Cover and Weighted Vertex Cover Problems.

Vertex Cover Problem (VCP)

Instance: A undirected graph $G=(V, E)$.

Objective: Find a set $C \subseteq V$ of minimum size such that for every $(i, j) \in E$ we have either $i \in C$ or $j \in C$.

Weighted Vertex Cover Problem (WVCP)

Instance: A undirected graph $G=(V, E)$, weights $w_{i} \geqslant 0 \forall i \in V$.

Objective: Find a Vertex Cover $C$ which minimizes $\sum_{i \in C} w_{i}$.

Several approximation algorithms have been proposed for the Unweighted and Weighted Versions of the Vertex Cover Problem. We list some of them in Table 1. We decided to implement these algorithms because of their simplicity and performance guarantees.

One of the first approximation algorithms in the literature for the Unweighted Vertex Cover problem is due to Gavril [8]. It is a greedy algorithm and is described next. Given an undirected graph $G=$ $(V, E)$, at each iteration of the algorithm, both nodes of a chosen edge $(i, j) \in E$ are selected to be in the cover, and the edges incident to nodes $i$ and $j$ are deleted from $E$. The algorithm terminates when the edge set $E$ is empty. Clearly the set created by the chosen nodes is a Vertex Cover of the graph $G$. The algorithm clearly runs in polynomial time. The performance guarantee of 2 follows from the facts that edges are only deleted from the edge set $E$ when they are covered, and at termination $E=\emptyset$.

Algorithm 1. Greedy algorithm for the Unweighted Vertex Cover.

Input: $G=(V, E)$

Output: Vertex cover $C$

$C \leftarrow \emptyset$;

while $E \neq \emptyset$ do

choose arbitrarily $(i, j) \in E$;

$C \leftarrow C \cup\{i, j\}$;

Delete $(i, j)$ from $E$ and all edges adjacent to $i$ and $j$;

end 
We now describe an algorithm for the Weighted Vertex Cover Problem. We first formulate the problem as an integer program.

$$
\begin{array}{ll}
\min & \sum_{i \in V} w_{i} x_{i} \\
\text { S.t. } & \\
& x_{i}+x_{j} \geqslant 1 \quad \forall(i, j) \in E, \\
& x_{i} \in\{0,1\} \quad \forall i \in V .
\end{array}
$$

The first constraint in the integer program says that for each edge $(i, j) \in E$ at least one of the nodes must be in the cover. The linear program (LP) of the integer program above is obtained by replacing constraints (4) by constraints $0 \leqslant x_{i} \leqslant 1 \forall i \in V$. By using this LP we derive the following algorithm.

Algorithm 2. Round algorithm for the weighted Vertex Cover Problem.

$$
\text { Input: } G=(V, E)
$$

Output: Vertex cover $C$

Solve the LP to get an optimal solution $x^{*}$;

$$
C \leftarrow \emptyset \text {; }
$$

for each $i \in V$ do

$$
\text { if } \begin{aligned}
x_{i}^{*} & \geqslant \frac{1}{2} \text { then } \\
C & \leftarrow C \cup\{i\} ;
\end{aligned}
$$

end

end

The Round algorithm produces a Vertex Cover and has performance guarantee of 2 .

We now turn to an algorithm based on the dual program of the LP. The dual of the LP above is

$$
\max \sum_{(i, j) \in E} y_{i, j}
$$

S.t.

$$
\begin{aligned}
& \sum_{j:(i, j) \in E} y_{i, j} \leqslant w_{i} \quad \forall i \in V \\
& y_{i, j} \geqslant 0 \quad \forall(i, j) \in E
\end{aligned}
$$

Then the dual-LP algorithm is as follows. 
Table 2

Approximation algorithms for the Set Covering Problem. The values of $f$ and $H_{n}$ will be defined later

\begin{tabular}{lll}
\hline Algorithm & $\begin{array}{l}\text { Performance } \\
\text { guarantee }\end{array}$ & Ref. \\
\hline Round & $f$ & {$[11]$} \\
Dual & $f$ & {$[11]$} \\
Primal-Dual & $f$ & {$[12]$} \\
Greedy & $H_{n}$ & {$[13]$} \\
\hline
\end{tabular}

Algorithm 3. Dual-LP algorithm for the weighted Vertex Cover Problem.

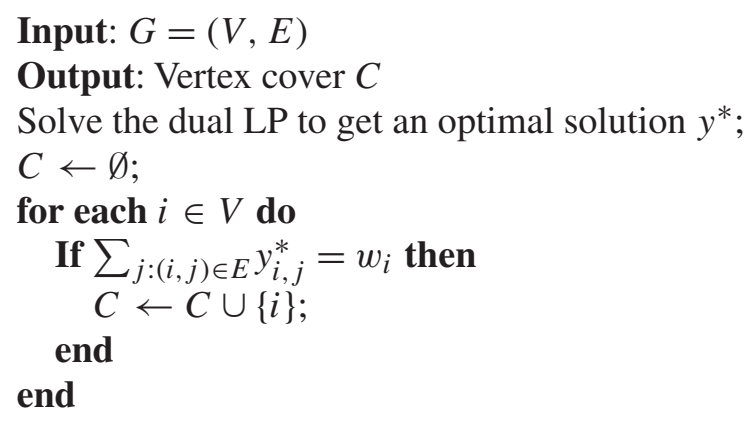

It is not difficult to show that the algorithm dual-LP returns a Vertex Cover and that its performance guarantee factor is 2 . Clearly the algorithm runs in polynomial time.

\subsection{Set Covering Problem}

In this section we define the Set Covering Problem and describe some of its approximation algorithms. The Set Covering Problem calls for a minimum cost family of subsets from $n$ given subsets, which together covers the entire ground set. Many real-world problems can be cast as a Set Covering Problem, for example, crew scheduling in airlines and driver scheduling in public mass transport. Often these problems are large in size and thus to try to find approximate solutions to them one will need to use some kind of heuristic technique. The study presented in this paper may be a guide to someone intending to use heuristic methods to derive approximate solutions to the Set Covering Problem. Next, we give a formal definition of the Set Covering Problem.

Set Covering Problem (SCP)

Instance: A ground set $E=\left\{e_{1}, \ldots, e_{n}\right\}$, subsets $S_{1}, \ldots, S_{m} \subseteq E$, and cost $w_{j}$ for each subset $S_{j}$.

Objective: Find a set $I \subseteq\{1, \ldots, m\}$ such that $\bigcup_{j \in I} S_{j}=E$ and $\sum_{j \in I} w_{j}$ is minimum.

Table 2 shows the approximation algorithms for the SCP we describe and test in this paper.

The first approximation algorithm we describe is based on an integer programming (IP) formulation for the SCP. To this end, define a variable $x_{j}$ for each subset $S_{j}$ such that if $j \in I$, then $x_{j}=1$, and if 
$j \notin I$, then $x_{j}=0$. An IP formulation to the SCP is as follows.

$\min \sum_{j=1}^{m} w_{j} x_{j}$

S.t.

$$
\begin{aligned}
& \sum_{j: e_{i} \in S_{j}} x_{j} \geqslant 1 \quad \forall e_{i} \in E, \\
& x_{j} \in\{0,1\} \quad j=1, \ldots, m .
\end{aligned}
$$

Define $f$ as

$$
f=\max _{1 \leqslant i \leqslant n}\left|\left\{j: e_{i} \in S_{j}\right\}\right|,
$$

i.e., $f$ is the maximum number of sets that contains a given element. From the linear relaxation of the IP formulation above we design the Round algorithm as follows.

Algorithm 4. Round algorithm.

Input: Finite ground set $E=\left\{e_{1}, e_{2}, \ldots, e_{n}\right\}$, subsets

$S_{1}, \ldots, S_{m} \subseteq E$, and cost $w_{j}$ for each subset $S_{j}$.

Output: Set covering $I$

Solve the linear program corresponding to the SCP to get an optimal solution $x^{*}$;

$I \leftarrow \emptyset$;

$$
\begin{aligned}
& \text { for each } S_{j} \text { do } \\
& \text { If } x_{j}^{*} \geqslant \frac{1}{f} \text { then } \\
& \qquad I \rightarrow I \cup\{j\} ; \\
& \text { end } \\
& \text { end }
\end{aligned}
$$

The Round algorithm is an $f$-approximation algorithm. We now design an algorithm based on the dual of the LP of the IP formulation for the SCP. The dual of the LP for the set covering is

$$
\begin{array}{ll}
\max & \sum_{i=1}^{n} y_{i} \\
\text { S.t. } & \\
& \sum_{i: e_{i} \in S_{j}} y_{i} \leqslant w_{j} \quad \forall S_{j}, \\
& y_{i} \geqslant 0 \quad \forall e_{i} \in E .
\end{array}
$$

Then the dual-LP algorithm is as follows. 
Algorithm 5. Dual-LP algorithm.

Input: Finite ground set $E=\left\{e_{1}, e_{2} \ldots, e_{n}\right\}$, subsets $S_{1}, \ldots, S_{m} \subseteq E$, and cost $w_{j}$ for each subset $S_{j}$.

Output: Set covering $I$

Solve the dual linear program to get an optimal solution $y^{*}$;

$I \leftarrow \emptyset$;

for each $S_{j}$ do

$$
\begin{aligned}
& \text { If } \sum_{i: e_{i} \in S_{j}} y_{i}^{*}=w_{j} \text { then } \\
& \quad I \leftarrow I \cup\{j\} ; \\
& \text { end }
\end{aligned}
$$

end

The dual-LP algorithm is an $f$-approximation algorithm. We now describe an algorithm that works like the Dual-LP but constructs its own dual solution rather than finding an optimal dual solution.

Algorithm 6. Primal-Dual algorithm.

Input: Finite ground set $E=\left\{e_{1}, \ldots, e_{n}\right\}$, subsets $S_{1}, \ldots, S_{m} \subseteq E$, and cost $w_{j}$ for each subset $S_{j}$.

Output: Set covering $I$

$I \leftarrow \emptyset$

for $i=1$ to $n$ do

$$
\bar{y}_{i} \leftarrow 0
$$

end

while $\exists e_{k}$ such that $e_{k} \notin \bigcup_{j \in I} S_{j}$ do

$l \leftarrow \operatorname{argmin}_{j: e_{k} \in S_{j}}\left\{w_{j}-\sum_{i: e_{i} \in S_{j}} \bar{y}_{i}\right\}$;

$\varepsilon_{l} \leftarrow w_{l}-\sum_{i: e_{i} \in S_{l}} \bar{y}_{i}$

$\bar{y}_{k} \leftarrow \bar{y}_{k}+\varepsilon_{l} ;$

$I \leftarrow I \cup\{l\}$;

end

The function argmin in Algorithm 6 returns the index that attains the minimum value.

The Primal-Dual returns a set covering and it constructs a dual feasible solution. This algorithm is an $f$-approximation algorithm.

Next, we present a greedy algorithm for the SCP. The basic idea is: go through the subsets $S_{j}$ and pick the set which will cover the most elements yet to be covered at the lowest cost. First define

$$
H_{n}=1+\frac{1}{2}+\frac{1}{3}+\cdots+\frac{1}{n} \cong \ln n .
$$

The greedy algorithm is an $H_{n}$-approximation algorithm. 
Algorithm 7. Greedy algorithm.

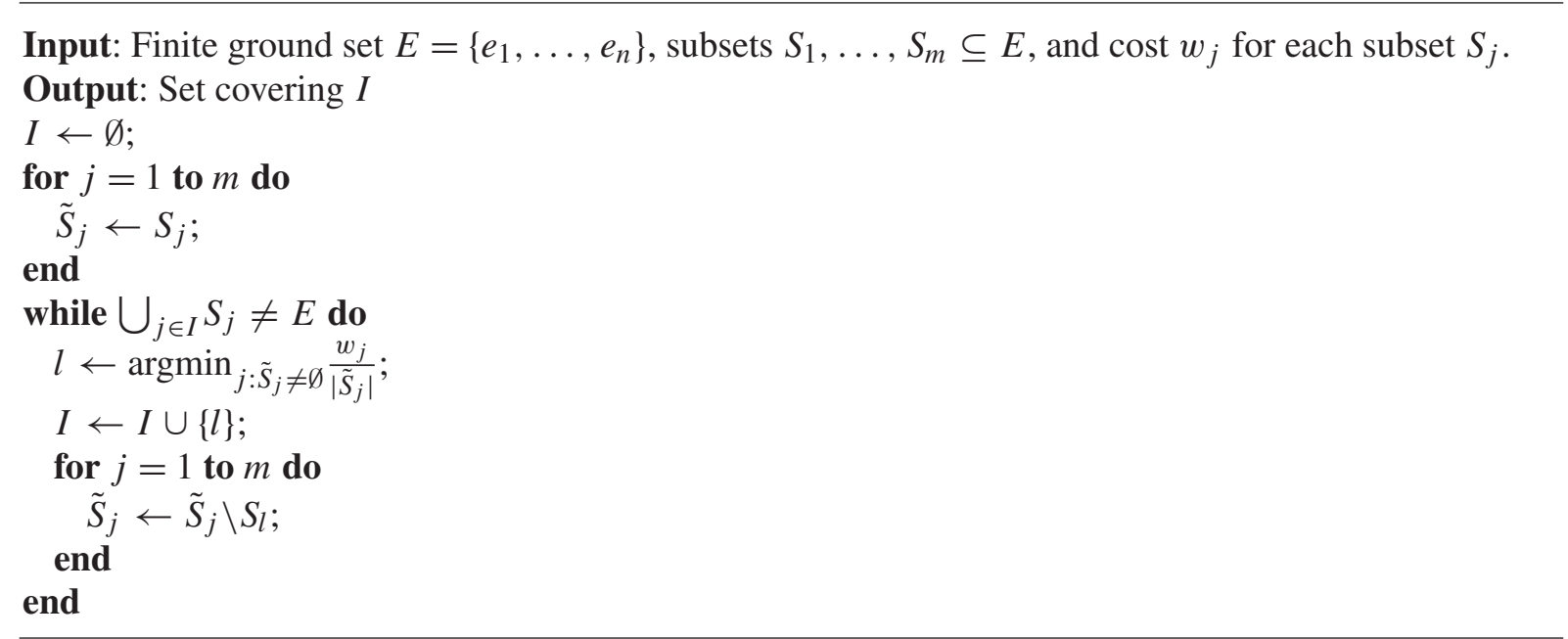

\section{Computational experiments}

In this section we present computational experiments carried out on instances of the Vertex Cover and Set Covering Problems.

The algorithms for the Vertex Cover Problem were tested on randomly generated instances, while the algorithms for the Set Covering Problem were tested on instances obtained from OR-LIBRARY [14].

All tests were executed on an AMD-Athlon PC with speed of $1.3 \mathrm{GHz}$ and $256 \mathrm{MB}$ of RAM under WindowsXP. The algorithms were implemented in Fortran PowerStation 4.0 language (Microsoft Developer Studio, 1995), and the Industrial LINDO/PC - Release 6.0 (Lindo System, Inc., 1996) software was used to solve linear and integer programs.

In the experiments we also report CPU times of all tested algorithms. On these cpu times we do not consider the time consumption to read an instance from file to RAM. We do not apply any kind of preprocessing on the instances. The cpu times are reported in the format 'hhmmss', i.e., hours, minutes, seconds. It is particularly noteworthy that computational times can be strongly influenced by implementation decisions. Due to this we used the same data structures on all algorithms, and we do not use any compile options.

Since we know the optimal solution value for each instance we tested, we can measure the quality of a solution derived by an algorithm by computing the ratio between them. That is, we define the quality measure ratio as value/optimum, where value is the value of a solution found by an algorithm, and optimum is the optimal solution value.

In the introduction section we set out to answer the question: "how far from the theoretically proven performance guarantee are the experimental results?". We use the ratio to respond that question. We note that the smaller the ratio, the further the experimental result is from the theoretically proven performance guarantee of an algorithm. 
Table 3

Averages and standard deviations of the ratio values for the Weighted Vertex Cover Problem

\begin{tabular}{lllll}
\hline Algorithm & Min & Average & Max & Std Dev \\
\hline Dual-LP & 1.19 & 1.33 & 1.58 & 0.10 \\
Greedy & 1.14 & 1.49 & 1.90 & 0.23 \\
Round & 1.00 & 1.51 & 1.85 & 0.27 \\
\hline
\end{tabular}

Table 4

Averages and standard deviations of the ratio values for the Unweighted Vertex Cover Problem

\begin{tabular}{lllll}
\hline Algorithm & Min & Average & Max & Std Dev \\
\hline Dual-LP & 1.15 & 1.25 & 1.43 & 0.06 \\
Greedy & 1.09 & 1.35 & 1.70 & 0.16 \\
Round & 1.24 & 1.58 & 2.00 & 0.21 \\
\hline
\end{tabular}

\subsection{Vertex Cover Problem}

The algorithms for the Vertex Cover Problem were tested on randomly generated instances. These instances were generated using the following procedure. We construct a random hamiltonian path over a node set of cardinality $n$. Then further edges are inserted into the graph at random. To each node we assign a random weight chosen uniformly in the interval $[1,100]$. We note that this graph is, by construction, connected. We generated 30 instances in this way, and duplicated these instances by changing all vertex weights to 1 . So, we test the algorithms on 60 instances.

In the process of generating those instances for the Vertex Cover Problem we used a random number generator which is an implementation of the multiplicative linear congruential generator [15], with parameters 16807 (multiplier) and $2^{31}-1$ (prime number).

Detailed computational experiments are shown in [16]. In this section we summarize those results. Tables 3 and 4 sum up information concerning the ratios.

Figs. 1 and 2 show the ratio values, plotted as points, for the Greedy, Dual-LP, and Round algorithms. For each algorithm their points are linked by a line. With these figures we just want to show that, for the set of instances we used, the Round algorithm is most of the time dominated by the other two algorithms. By dominated algorithm we mean the algorithm never produced better solutions than some other algorithm.

\subsection{Set Covering Problem}

In this section we present computational experiments carried on instances of the Set Covering Problem. We tested 60 instances. Thirty of these instances were obtained from OR-LIBRARY [14]. By replacing the weights to 1 on these instances, we generated 30 instances to the Unweighted SCP.

Detailed computational experiments are given in [16]. In this section we summarize those results. Tables 5 and 6 sum up the information concerning the ratios. 


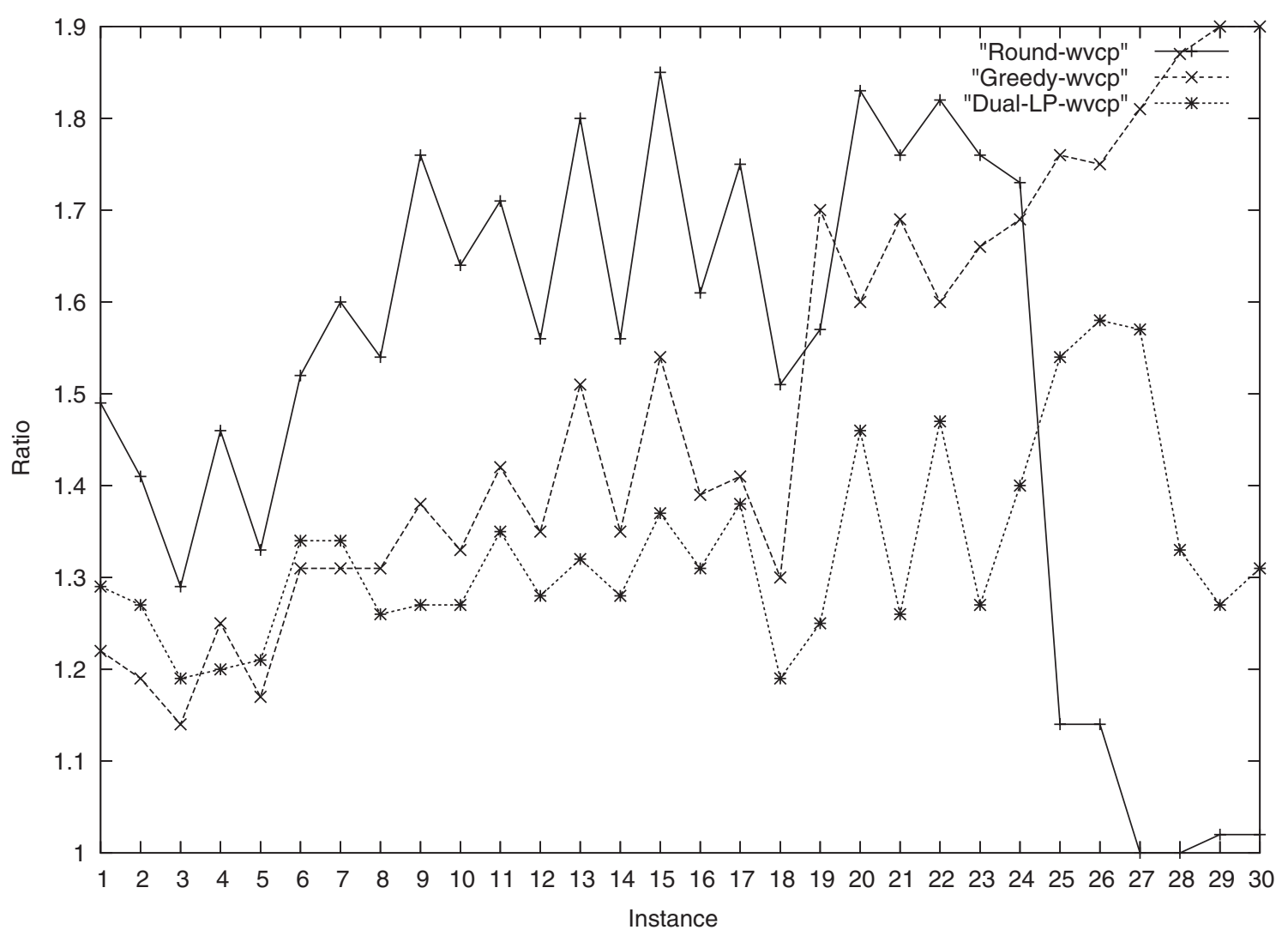

Fig. 1. Ratio information of the Greedy, Round and Dual-LP algorithms for the Weighted Vertex Cover Problem. Each $x$-coordinate in the figure corresponds to an instance.

Figs. 3 and 4 show the ratio values, plotted as points, for the Round, Dual-LP, Primal-Dual, and Greedy algorithms. The points corresponding to an algorithm are joined by a line. From Figs. 3 and 4 we see that the Greedy algorithm virtually dominates the other methods.

\section{Discussion}

\subsection{Vertex Cover Problem}

For the unweighted and weighted versions of the VCP, the Greedy and the Dual-LP algorithms performed well on all of the tested instances.

Consider the unweighted VCP. For all instances, the Dual-LP algorithm performed better than the Round algorithm. The Greedy algorithm was faster than the Round and the Dual-LP algorithms. On the 60 instances, none of the algorithms found an optimal solution, but the empirical performances of the Greedy and the Dual-LP are much better than their proven performance guarantees. 


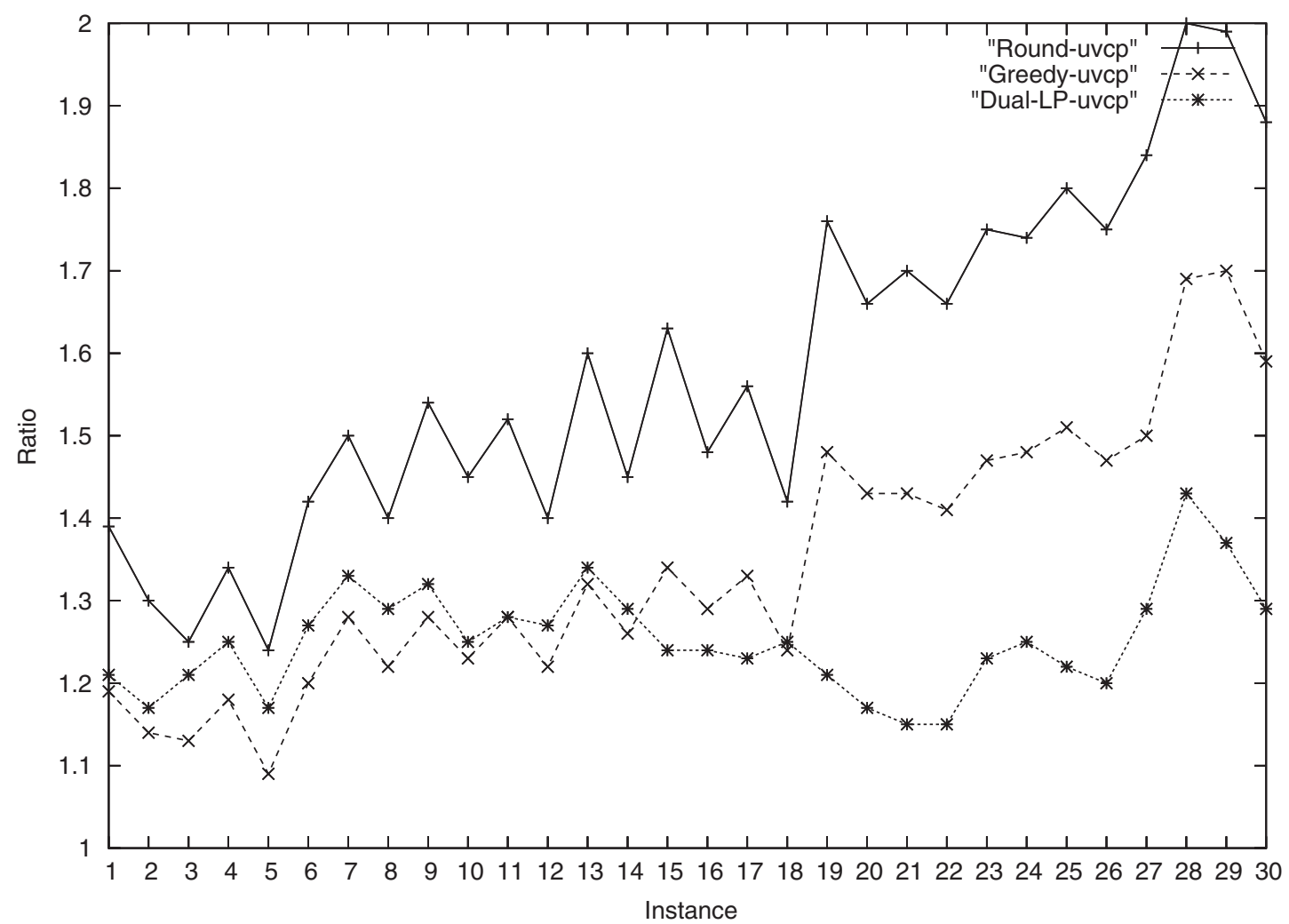

Fig. 2. Ratio information of the Greedy, Round and Dual-LP algorithms for the Unweighted Vertex Cover Problem. Each $x$-coordinate in the figure corresponds to an instance.

Consider the weighted VCP. The Dual-LP, the Greedy, and the Round algorithms deviated, on average, by $33 \%, 49 \%$, and $51 \%$ from the optimal solution value (See Table 3). For the unweighted VCP, the Greedy deviated by $35 \%$ from the optimum value, the Dual-LP deviated by $25 \%$, and the Round deviated by $58 \%$ (See Table 4 ).

Instance 28 of the unweighted VCP (see Fig. 2) represents a worst case for the Round algorithm, since its ratio was of 2 . This algorithm also performs badly on the instance number 29 for the same problem. We infer from that figure that the Round algorithm is dominated by the Greedy and Dual-LP algorithms.

Figs. 1 and 2 also show the effect of weighted versus unweighted versions of the Vertex Cover Problem relative to the performance of the algorithms. From both figures wee see a superior performance of the Dual-LP. This can also be noticed from the results in Tables 3 and 4.

We have noticed that the Round algorithm outperformed the Greedy and the Dual-LP for instances at which the linear relaxation of the model (1)-(4) is almost integer. That is, the solution vector $x$ has few components $x_{i}$ with fractional values. When this is not the case, the Round algorithm performed badly.

\subsection{Set Covering Problem}

For the unweighted and weighted versions of the SCP, the Greedy algorithm performed extremely well on all of the tested instances. All algorithms were very fast. On the 60 instances, the optimum value was 
Table 5

Averages and standard deviations of the ratio values for the Weighted Set Covering Problem

\begin{tabular}{lcccc}
\hline Algorithm & Min & Average & Max & Std Dev \\
\hline Greedy & 1.03 & 1.13 & 1.24 & 0.05 \\
Dual-LP & 1.18 & 1.55 & 2.23 & 0.27 \\
Primal-Dual & 1.21 & 1.61 & 2.28 & 0.30 \\
Round & 1.00 & 1.98 & 3.19 & 0.65 \\
\hline
\end{tabular}

Table 6

Averages and standard deviations of the ratio values for the Unweighted Set Covering Problem

\begin{tabular}{lcccc}
\hline Algorithm & Min & Average & Max & Std Dev \\
\hline Greedy & 1.00 & 1.07 & 1.18 & 0.05 \\
Dual-LP & 1.57 & 1.93 & 2.42 & 0.20 \\
Primal-Dual & 1.00 & 2.42 & 3.23 & 0.69 \\
Round & 1.49 & 4.24 & 8.64 & 2.07 \\
\hline
\end{tabular}

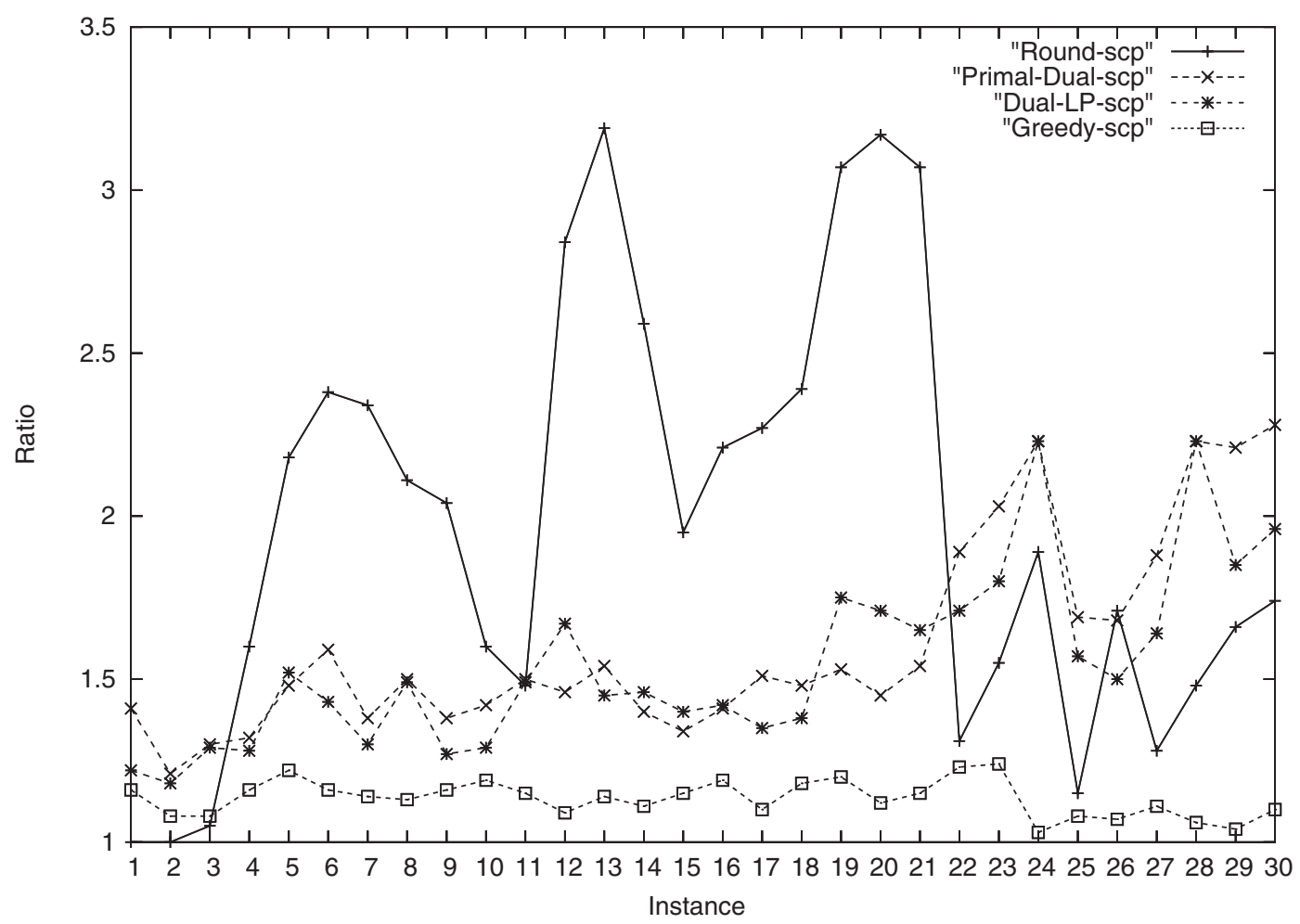

Fig. 3. Ratio information of the Round, Dual-LP, Primal-Dual, and Greedy algorithms for the Weighted Set Covering Problem. Each $x$-coordinate in the figure corresponds to an instance. 


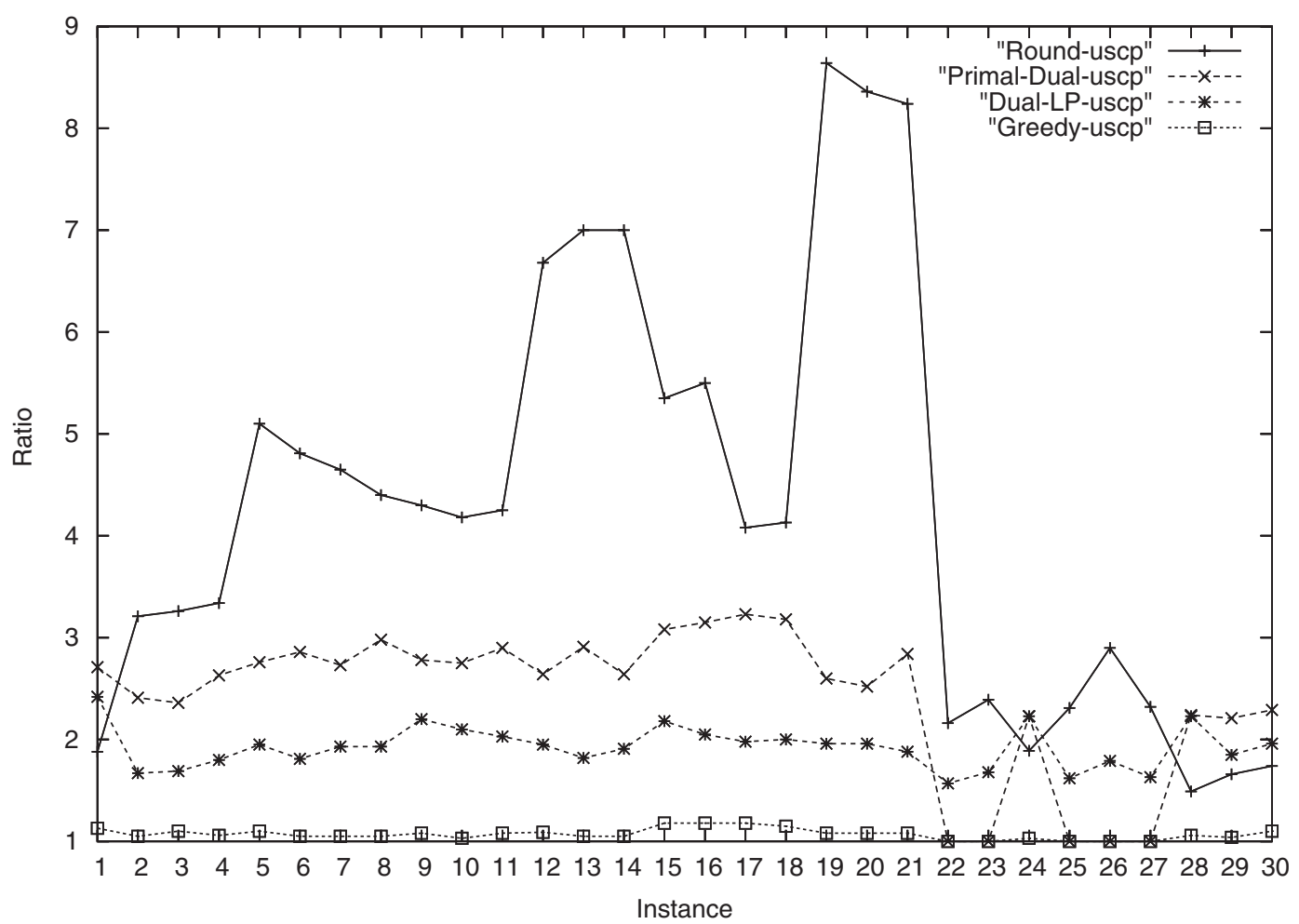

Fig. 4. Ratio information of the Round, Dual-LP, Primal-Dual, and Greedy algorithms for the Unweighted Set Covering Problem. Each $x$-coordinate in the figure corresponds to an instance.

found 2 times by the Round algorithm, 5 times by the Greedy algorithm, and 5 times by the Primal-Dual algorithm.

Considering the unweighted SCP, on average the Greedy deviated by $7 \%$ from the optimum value, the Dual-LP deviated by $93 \%$, the Primal-Dual deviated by $142 \%$, and the Round deviated by $324 \%$ (See Table 6). With respect to the weighted SCP, on average the Greedy deviated by $13 \%$ from the optimum value, the Dual-LP deviated by 55\%, the Primal-Dual deviated by $61 \%$, and the Round deviated by $98 \%$ (See Table 5).

We see from Fig. 3 the Primal-Dual and the Dual-LP had almost the same performance. In Fig. 4 we notice that the Dual-LP outperforms the Primal-Dual for almost all instances. From these figures we clearly see the influence of the weighted versus unweighted versions relative to the performance of the algorithms. The Greedy algorithm seems to be less sensitive to the effect of weighted versus unweighted, while the Round algorithm is greatly influenced by it.

\section{Concluding remarks}

This paper investigated the empirical performance of approximation algorithms for the Vertex Cover and Set Covering Problems. We implemented seven approximation algorithms altogether. 
For the VCP we implemented three algorithms: Greedy, Round, and Dual-LP. The overall best algorithm we tested was the Greedy algorithm, followed by the Dual-LP and the Round, in this order. The Greedy algorithm was the fastest one and on average its empirical performance was close to the unity.

For the SCP we implemented four algorithms: Round, Dual-LP, Primal-Dual, and Greedy. All algorithms were fast, and the Greedy algorithm performed well on all of the instances. On average, the Round and Primal-Dual algorithms performed worse than the Greedy and Dual-LP algorithms.

We remark that the proven performance guarantees of all tested algorithms did not forecast well the empirical performance.

For the tested algorithms, the computational experiments demonstrated that approximation algorithms based on the dual program of a linear program achieved better experimental results than the approximation algorithms using the primal problem. To try to have a better understanding of the empirical performance we plan as future research the development of a tool which would facilitate the experimental analysis of algorithms.

\section{References}

[1] Grossman T, Wool A. Computational experience with approximation algorithms for the set covering problem. European Journal of Operational Research 1997;101.

[2] Barr RS, Golden BL, Kelly JP, Resende MGC, Stewart WR. Designing and reporting on computational experiments with heuristic methods. Journal of Heuristics 1995;1:9-32.

[3] Johnson DS. A theoretician's guide to the experimental analysis of algorithms. In: Goldwasser M, Johnson DS, McGeoch CC, editors. Proceedings of the fifth and sixth DIMACS implementation challenges. Providence, RI: American Mathematical Society; 2002.

[4] Sherali HD, Rios M. An air force crew allocation and scheduling problem. Journal of the Operations Research Society $1984 ; 35$.

[5] Bhattacharyya SS, Sundararajan S. Resynchronization for multiprocessor DSP systems. IEEE Transactions on Circuits-I: Fundamental Theory and Applications 2000;47(11).

[6] Fischetti M, Caprara A, Toth P, Vigo D. Modeling and solving the crew rostering problem. Operations Research $1998 ; 46$.

[7] Woodyatt LR, Stott KL, Wolf FE, Vasko FJ. An application combining set covering and fuzzy sets to optimally assign metallurgical grades to customer orders. Fuzzy Sets and Systems 1993;53.

[8] Gavril F. Private Communication cited in [16], 1974.

[9] Hochbaum DS. Efficient bounds for the stable set, vertex cover and set packing problems. Discrete Applied Mathematics 1983;6:243-54.

[10] Nemhauser GL, Trotter Jr LE. Vertex packings: structural properties and algorithms. Mathematical Programming $1975 ; 8: 232-48$.

[11] Hochbaum DS. Approximation algorithms for the set covering and vertex cover problems. SIAM Journal on Computing 1982;11(3):555-6.

[12] Bar-Yehuda R, Even S. A linear time approximation algorithm for the weighted vertex cover problem. Journal of Algorithms 1981;2:198-203.

[13] Chvatal V. A greedy-heuristic for the set covering problem. Mathematics of Operations Research 1979;4:233-5.

[14] Beasley JE. OR-LIBRARY - a combinatorial optimization problem library. Website: http://mscmga.ms.ic.ac.uk /jeb/orlib/scpinfo.html.

[15] Park S, Miller K. Random number generators: good ones are hard to find. Communications of the ACM 1988;31: 1192-201.

[16] Gomes FC, Meneses CN, Pardalos PM, Viana GVR. computational results of approximation algorithms for the vertex cover and set covering problems. Manuscript. 2005. 


\section{Further Reading}

[17] Bomze IM, Budinich M, Pardalos PM, Pelillo M. The maximum clique problem. In: Du D-Z, Pardalos PM, editors. Handbook of combinatorial optimization, Suppl. vol. A. Dordrecht: Kluwer Academic Publishers; 1999.

[18] Abello J, Pardalos PM, Resende M. On maximum clique problems in very large graphs. External memory algorithms. In: Abello JM, Vitter JS, editors. DIMACS series, vol. 50. Providence, RI: American Mathematical Society; 1999.

[19] Hoffman KL, Padberg M. Solving airline crew scheduling problems by branch-and-cut. Management Science 2003;39(6):657-82.

[20] Garey MR, Johnson DS. Computers and intractability: a guide to the theory of NP-completeness. San Francisco, CA: Freeman; 1979.

[21] Busygin S, Butenko S, Pardalos PM. A heuristic for the maximum independent set problem based on optimization of a quadratic over a sphere. Journal of Combinatorial Optimization 2002;6(3):287-97.

[22] Butenko S, Pardalos PM, Sergienko I, Shylo V, Stetsyuk P. Finding maximum independent sets in graphs arising from coding theory. In: Symposium on applied computing. Madrid, Spain: ACM Press; 2002. p. 542-6. 